\title{
Holiday heart syndrome: do not drink during this holiday! and other updates on recent autonomic research
}

\author{
Mitchell G. Miglis ${ }^{1} \cdot$ Nicholas Larsen $^{1} \cdot$ Srikanth Muppidi ${ }^{1,2}$
}

Received: 5 July 2021 / Accepted: 5 July 2021 / Published online: 9 July 2021

(c) Springer-Verlag GmbH Germany, part of Springer Nature 2021

Keywords Alcohol $\cdot$ Familial autonomic ganglionopathy $\cdot$ Cholinergic $\cdot$ Acetylcholine $\cdot$ Heart rate variability $\cdot$ SUDEP

\begin{abstract}
"Holiday heart syndrome" is a term used to describe cardiac arrhythmias caused by excessive alcohol intake in otherwise healthy individuals. There is evidence that acute alcohol consumption leads to sinus tachycardia along with impairment of the physiologic respiratory sinus arrhythmia, suggesting an imbalance between the parasympathetic and sympathetic autonomic nervous systems [3]. Brunner and colleagues published in Scientific Reports their experience using novel electrocardiogram (ECG)-based measures to understand how acute ethanol intake affects the cardiac autonomic nervous system [4]. The novel electrocardiogram (ECG)-based measures used in this study include deceleration capacity (DC) and periodic repolarization dynamics (PRD). DC is a robustly validated measure of all deceleration-related oscillations of heart rate and is a marker of heart rate variability (HRV), which is, in turn, a measure of cardiovagal tone. PRD quantifies dynamic properties of the T-wave that are thought to be caused by phasic activation of the efferent sympathetic nervous system. Impaired DC is a predictor of late mortality after myocardial infarction [2], while increased PRD is associated with higher risk for ventricular arrhythmias and sudden death in patients with heart failure [9].

The authors enrolled eight healthy men and seven healthy women with a mean age of 28 years. Participants with a history of heavy drinking, cardiovascular disease, respiratory disease, cerebrovascular disease, infectious disease, alcohol abstinence, and those taking medications were excluded. Alcohol was administered intravenously using a
\end{abstract}

Srikanth Muppidi

muppidis@stanford.edu

Stanford Medical Center, Palo Alto, CA, USA

2 Stanford Neurosciences Health Center, 213 Quarry Road, 2nd Floor, Palo Alto, CA 94304, USA
$7 \%$ ethanol $/ 5 \%$ glucose solution until patients reached a target breath alcohol concentration of $0.50 \mathrm{mg} / \mathrm{l}$ (equivalent to $0.100 \mathrm{~g} / \mathrm{dl}$ serum alcohol level; to achieve this level, a 70-kg man would have to consume approximately four standard drinks of alcohol in $1 \mathrm{~h}$ ). All individuals then underwent high-resolution digital 30-min ECG in a supine and resting position. Measurements were obtained before alcohol administration, at the time of maximum alcohol concentration, and after breath alcohol concentrations dropped to $0.05 \mathrm{mg} / \mathrm{l}$. Heart rate, PRD, DC, and HRV with deep breathing were assessed using customized software.

The results showed that the median pre-alcohol PRD level was $0.85 \mathrm{deg}^{2}$, while the median PRD level at maximum alcohol concentration significantly increased to 1.92 $\operatorname{deg}^{2}(p=0.001)$. From the maximum alcohol concentration, the median post-alcohol PRD level (at $0.05 \mathrm{mg} / \mathrm{l}$ alcohol level) decreased to $1.44(p=0.09)$. When compared to the median pre-alcohol levels, the median post-alcohol PRD level was significantly elevated $(p=0.042)$. Compared to the maximum alcohol concentration, the post-alcohol DC level increased significantly to $8.66(p=0.041)$. The post-alcohol DC level did not differ significantly from the pre-alcohol level $(p=0.24)$. Standard measures of HRV decreased from baseline to maximal alcohol concentration and slightly increased as alcohol levels returned towards normal. The mean heart rate increased from baseline to maximal alcohol concentration and remained elevated.

Previous studies have shown that acute alcohol intake leads to an increase of sinus tachycardia and a reduction of respiratory sinus arrhythmia, which is suggestive of an imbalance in the autonomic nervous system [3]. However, most studies investigating the effects of alcohol intake on the autonomic nervous system have used standard HRV measures, which are influenced by both the parasympathetic and sympathetic nervous systems. This was the first study to show that acute alcohol intake leads to a significant 
increase of PRD and reduction in DC at the time of maximal alcohol concentration. These findings suggest that acute alcohol intake leads to an increase in sympathetic activity and a decrease in parasympathetic activity, resulting in an autonomic imbalance that may trigger the arrhythmias featuring the "holiday heart syndrome."

Limitations of this study include a small sample size and non-continuous data monitoring (the ECGs were only analyzed at specific time points). It would have been interesting to include additional time points to evaluate how long it took for the DC and PRD levels to return to baseline. Future studies should focus on determining the kinetics of alcohol-related changes in the autonomic nervous system and measuring the effect that chronic alcohol intake has on DC and RPD. But, in any case, the findings by Brunner and colleagues are a good reason to stay sober this holiday!

\section{Familial autonomic ganglionopathy: a new genetic autonomic disorder on the block}

Acetylcholine receptors (AChR) are critical for the optimal transmission of autonomic signals. Auto-antibodies blocking the muscarinic $\mathrm{M}_{3}$ acetylcholine receptors in organs and tissues cause postganglionic cholinergic dysautonomia, a syndrome featuring generalized exocrine gland failure [7]; whereas auto-antibodies blocking ganglionic nicotinic acetylcholine receptors cause generalized and severe autonomic failure referred to as autoimmune autonomic ganglionopathy (AAG) [6]. When AAG was originally described, it was considered by some as an "autonomic" myasthenia [5]; not a bad moniker, as many patients with autoimmune myasthenia gravis also have anti-nicotinic receptor antibodies. While the comparison might have been overly simplistic, it did provide a benchmark for other non-autoimmune autonomic ganglionopathies.

Similar to congenital or genetic forms of myasthenia gravis, Shibao and colleagues have recently described a rare familial form of autonomic ganglionopathy, which they recently published in Neurology [10]. This publication details the effort by the authors in three patients with familial autonomic ganglionopathy. Two patients were siblings from one family and another patient was from a different unrelated family. All three patients were evaluated formally at their center and completed standardized autonomic testing as well as various pharmacological studies. All patients presented with significant orthostatic symptoms from a young age along with symptoms of slow gastrointestinal motility and visual symptoms. All patients were also documented to have neurogenic orthostatic hypotension $(\mathrm{nOH})$ with a systolic blood pressure drop of $26-58 \mathrm{mmHg}$ when standing up. On autonomic testing, Valsalva indices were abnormal with prolonged pressure recovery time suggesting a sympathetic failure. The cardiovagal evaluation with heart rate variability was abnormal for two patients from the same family. All patients had reduced supine and standing norepinephrine levels. Finally, all had abnormal pupillary reflexes and additional ocular abnormalities.

The two siblings from the same family were evaluated in 1995 and again more recently and another patient was evaluated in 2019. Genetic testing with exome sequencing was performed with the help of the Undiagnosed Disease Network and pathogenic mutations were found in the affected family members in the gene for acetylcholine receptor neuronal nicotinic alpha-3 subunit. Extensive clinical evaluation, autonomic testing, pharmacological and genetic testing suggest that familial autonomic ganglionopathy is caused by mutations in the CHRNA3 gene. As a consequence of these mutations, patients develop autonomic failure at a very young age. In addition to $\mathrm{nOH}$, patients have abnormal pupillary light reflexes with poorly reactive small pupils, ptosis, lightly colored irises, and reduced tear production. Interestingly one patient who was treated with pyridostigmine had a significant improvement in the degree of symptomatic hypotension as well as standing time, again supporting that impaired autonomic ganglionic transmission is a primary dysfunction in these patients.

While the authors are to be commended for their work to understand this rare syndrome with deep phenotypic evaluation and help us understand the underlying genetic basis for the syndrome, we wish there was further evaluation with different pharmacotherapeutic agents including pyridostigmine in all subjects for a longer period to see if clinical benefit persists. To a practicing autonomic physician, this publication highlight that the occurrence of congenital autonomic deficits suggests a genetic disorder, and adds to other, recently described genetic mutations causing autonomic dysfunction $[8,11]$. This is also relevant for therapeutic reasons as many of these genetic mutations could be amenable to gene therapies, hopefully in the not-so-distant future.

\section{Abnormal heart rate variability during hyperventilation: a biomarker of sudden unexpected death in epilepsy (SUDEP)?}

Sudden unexpected death in epilepsy (SUDEP) is the most feared complication of epilepsy, with prevalence estimates of as high as 1 in 200 patients per years in those with drugresistant epilepsy. The common occurrence of SUDEP during sleep and its association with abnormalities in heart rate variability (HRV) and arrhythmias suggest a role of autonomic dysfunction in the pathogenesis [1]. In a recent article published in Neurology, Szurhaj and colleagues performed a retrospective case-control study to further evaluate this 
theory, using HRV analysis of hyperventilation periods during electroencephalogram (EEG) [12]. SUDEP cases were selected from the cohort of the French Sentinel Mortality Epilepsy Network, and age and gender-matched controls with epilepsy were selected from several other databases.

The authors reviewed electrocardiogram (ECG) tracings before, during, and after hyperventilation. They calculated EEG spectral power and measures of HRV including lowfrequency (LF) power $(0.04-0.15 \mathrm{~Hz}$, a correlate of sympathetic tone), and high frequency (HF) power $(0.15-0.4 \mathrm{~Hz}$, a correlate of parasympathetic tone), the standard deviation of normal-to-normal RR intervals (SDNN, sympathetic and parasympathetic, a measure of global heart health), and the root mean square of successive RR-interval differences (RMSSD, parasympathetic time-domain measure). Patients with diabetes or known cardiac comorbidities were excluded.

Twenty cases of SUDEP were identified. At rest, there was no difference in HRV in those with SUDEP compared to controls. During hyperventilation, however, SUDEP patients had no significant changes in heart rate (HR), whereas in controls HR increased during hyperventilation and then returned to the baseline on average two minutes after hyperventilation. There was also a statistically significant decrease in RMSSD in controls after hyperventilation, followed by a return to baseline 4 min later; this was not seen in the SUDEP group. While there was a slight increase in the LF/ HF ratio in the SUDEP group, this was not statistically significant after correcting for multiple variables. The authors were able to determine that the change in HR between the end of hyperventilation and four minutes later predicted the occurrence of SUDEP with a sensitivity of $85 \%$ and a specificity of $75 \%$.

The limitations of the study include its small sample size, the use of medications that could potentially affect HRV, as well as other cofounding factors that were not accounted for, such as volume status, time of day, and the presence of cardiovascular deconditioning. Nevertheless, these findings support the growing body of literature that suggests cardiac autonomic dysfunction is a risk factor for SUDEP, and future studies are necessary to better understand the role of autonomic imbalance in this fatal condition.

Funding None.

\section{Declarations}

Conflict of interest None.

\section{References}

1. Barot N, Nei M (2019) Autonomic aspects of sudden unexpected death in epilepsy (SUDEP). Clin Auton Res 29:151-160

2. Bauer A, Kantelhardt JW, Barthel P, Schneider R, Makikallio T, Ulm K, Hnatkova K, Schomig A, Huikuri H, Bunde A, Malik M, Schmidt G (2006) Deceleration capacity of heart rate as a predictor of mortality after myocardial infarction: cohort study. Lancet 367:1674-1681

3. Brunner S, Herbel R, Drobesch C, Peters A, Massberg S, Kaab S, Sinner MF (2017) Alcohol consumption, sinus tachycardia, and cardiac arrhythmias at the Munich Octoberfest: results from the Munich Beer Related Electrocardiogram Workup Study (MunichBREW). Eur Heart J 38:2100-2106

4. Brunner S, Winter R, Werzer C, von Stulpnagel L, Clasen I, Hameder A, Stover A, Graw M, Bauer A, Sinner MF (2021) Impact of acute ethanol intake on cardiac autonomic regulation. Sci Rep 11:13255

5. Drachman DB (2003) Autonomic "myasthenia": the case for an autoimmune pathogenesis. J Clin Invest 111:797-799

6. Golden EP, Vernino S (2019) Autoimmune autonomic neuropathies and ganglionopathies: epidemiology, pathophysiology, and therapeutic advances. Clin Auton Res 29:277-288

7. Palma JA, Gupta A, Sierra S, Gomes I, Balgobin B, NorcliffeKaufmann L, Devi LA, Kaufmann H (2020) Autoantibodies blocking M3 muscarinic receptors cause postganglionic cholinergic dysautonomia. Ann Neurol 88:1237-1243

8. Palma JA, Yadav R, Gao D, Norcliffe-Kaufmann L, Slaugenhaupt S, Kaufmann H (2021) Expanding the genotypic spectrum of congenital sensory and autonomic neuropathies using whole-exome sequencing. Neurol Genet 7:e568

9. Rizas KD, McNitt S, Hamm W, Massberg S, Kaab S, Zareba W, Couderc JP, Bauer A (2017) Prediction of sudden and non-sudden cardiac death in post-infarction patients with reduced left ventricular ejection fraction by periodic repolarization dynamics: MADIT-II substudy. Eur Heart J 38:2110-2118

10. Shibao C, Joos K, Phillips JA 3rd, Cogan J, Newman JH, Hamid R, Meiler J, Capra J, Sheehan J, Vetrini F, Yang Y, Black B, Diedrich A, Roberston D, Biaggioni I (2021) Familial autonomic ganglionopathy caused by rare CHRNA3 genetic variants. Neurology. https://doi.org/10.1212/WNL.0000000000012143

11. Shibao CA, Garland EM, Black BK, Mathias CJ, Grant MB, Root AW, Robertson D, Biaggioni I (2020) Congenital absence of norepinephrine due to CYB561 mutations. Neurology 94:e200-e204

12. Szurhaj W, Leclancher A, Nica A, Perin B, Derambure P, Convers P, Mazzola L, Godet B, Faucanie M, Picot MC, De Jonckheere J (2021) Cardiac autonomic dysfunction and risk of sudden unexpected death in epilepsy. Neurology 96:e2619-e2626 